\title{
OPTIMIZACIÓN DE LA SOLDADURA EN CONSTRUCCIÓN NAVAL EN EL PERÚ
}

\section{OPTIMIZATION OF WELDING SHIPBUILDING IN PERU}

\author{
Hugo Eliseo Gamarra Chinchay ${ }^{1}$, Anwar Julio Yarin Achachagua ${ }^{2}$, Yasser Hipólito Yarin \\ Achachagua $^{3}$, Mierwen Palacios Aranda ${ }^{4}$
}

\begin{abstract}
RESUMEN
El presente trabajo estudia la productividad de la soldadura en construcción naval, partiendo de un análisis de la producción en nuestro medio pasando a presentar algunas técnicas o alternativas para incrementar la productividad de la soldadura en construcción naval. Comenzaremos describiendo algunos conocimientos básicos de soldadura, productividad y el como determinar la productividad de soldadura en la construcción naval, además del porque del análisis de la productividad de la soldadura en nuestro medio. Asimismo se exponen algunos factores que van a influir en la producción de la soldadura, como son el rendimiento por fusión, la selección de los materiales, el tipo de acero, el tipo de procedimiento, etc. Por otro lado, se presentan los datos estadísticos tomados en la zona de trabajo que servirán para medir la productividad de la soldadura en función de los kilogramos de metal depositado y el rendimiento por fusión de varios tipos de electrodos. Finalmente, se presenta la propuesta para incrementar la productividad de la soldadura en nuestro medio en base a los problemas observados y los cálculos realizados, presentando además dos procedimientos de soldadura que pueden ser aplicados en nuestro medio, y un método que facilitará la solución de los problemas que son detectados en el astillero.
\end{abstract}

Palabras clave.- Construcción naval, Procedimientos, Astilleros.

\begin{abstract}
The present work studies the productivity of the weld in naval construction, starting off of an analysis of the production in our means happening to present some techniques or alternatives to increase the productivity of the weld in naval construction. We will begin describing some basic knowledge of weld, productivity and like determining the productivity of weld in the naval construction, in addition to because of the analysis of the productivity of the weld in our means. Also some factors are exposed that are going to influence in the production of the weld, as they are the yield by fusion, the selection of the materials, the type of steel, the type of procedure, etc. On the other hand, the statistical data taken in the zone from work appear that will serve to measure the productivity of the weld based on the kilograms of deposited metal and the yield by fusion of several types of electrodes. Finally, the proposal appears to increase the productivity of the weld in our means on the basis of the observed problems and the made calculations, presenting in addition two welding procedures that can be applied in our means, and a method that will facilitate the solution of the problems that are detected in the shipyard.
\end{abstract}

Keywords.-. Shipbuilding, Procedures, Ahipyards.

${ }^{1}$ Mg. Ing. De la Facultad de Ingeniería Mecánica de la Universidad Nacional de Ingeniería, ${ }^{2}$ Ing. De la Facultad de Ingeniería Mecánica de la Universidad Nacional de Ingeniería, ${ }^{3}$ Ing. De la Facultad de Ingeniería Mecánica de la Universidad Nacional de Ingeniería, ${ }^{4}$ Ing. De la Facultad de Ingeniería Mecánica de la Universidad Nacional de Ingeniería. 


\section{INRODUCCION}

\section{CONCEPTO DE PRODUCTIVIDAD}

En primer lugar daremos una definición de productividad en forma general, para luego establecer una relación con la soldadura; de igual manera definiremos las unidades adecuadas para medir la productividad de la soldadura en construcción naval.

Se entiende por productividad a la relación entre el producto terminado y los recursos necesarios para su fabricación, sean humanos o no, donde el reto más grande para el factor humano es cómo economizar el uso de estos recursos para un producto determinado, es decir, cómo elevar en forma tangible el factor total de productividad. Esto envuelve innovaciones en los métodos de producción creando, adaptando y aplicando nuevas tecnologías con el fin de obtener mejores resultados para un mismo costo real.

Ahora vamos a definir la productividad en la soldadura, siendo conceptuada como la relación entre el valor de los resultados de los trabajos de soldadura y todos los costos que son necesarios para su realización, por lo cual, si el producto soldado esta bajo las normas de control de calidad, podemos afirmar que: el valor del resultado de los trabajos de soldadura es un número determinado de uniones soldadas o bien una cantidad fija de metal fundido sobre la unión. Para medir la productividad de la soldadura intervienen cuatro elementos fundamentales, éstos son:

1. Costo de la mano de obra.

2. Costos de los consumibles de soldadura.

3. Costos de la energía consumida para la soldadura.

4. Amortización de los equipos o máquinas para soldar.

De todos los costos necesarios para la soldadura, el más importante es la mano de obra en razón de que cada vez los salarios son más elevados, lo que origina un incremento en los gastos generales del astillero.

Por lo expuesto, si fundimos el metal depositado en menor tiempo estamos hablando de ahorro hombres/hora, siendo favorable para el astillero, pues, representa una reducción en los gastos generales de fabricación del buque permitiendo con esto participar en el mercado de la construcción naval con mayores oportunidades de ganar un concurso de ofertas.

$$
\begin{aligned}
& \mathrm{N}=\frac{\text { cantidad de metal depositado }}{\cos \text { to mano obra }+\cos \text { to combustible }+} \\
& \text { cos to energía }+\cos \text { to de anortización }
\end{aligned}
$$

Para establecer el rendimiento de un proceso de soldadura, se debe medir la productividad de la soldadura en función de la cantidad de metal depositado en una unidad de tiempo (kg.m.d/hr), al expresar el rendimiento de un proceso según la relación anterior encontramos que el aspecto más importante es el tiempo de fusión.

\section{COSTO DEL KILOGRAMO DE METAL DEPOSITADO EN SOLDADURA}

Para calcular el costo de las uniones soldadas en nuestro medio donde la soldadura Manual es el procedimiento más utilizado, existe una forma rápida de realizar y es en función de la cantidad de electrodos consumidos, permitiendo así tener una idea parcial del costo real de las uniones soldadas, a pesar de que algunos factores no son considerados, tales como: tiempo de fusión de cada electrodo, kilogramo de metal depositado sobre la unión y otros. Por lo tanto, si queremos conocer el gasto real del astillero en cuanto a las uniones soldadas, debemos calcular necesariamente el verdadero costo del kilogramo de metal depositado.

Los factores que intervienen son:

- Tf: tiempo de fusión de un kg.m.d (hr)

- Te: tiempos específicos del procedimiento (hr)

- Ta: tiempos auxiliares del procedimiento (hr)

- Ct: costo de una hora del soldador $(\$ / \mathrm{hr})$

- Cc: costo de consumibles $(\$ / \mathrm{hr})$

- Cm: amortización de la máquina de soldar $(\$ / \mathrm{kg})$

- Ce: costo de energía consumida (\$/kw.hr)

Para calcular el costo por 1 kg.m.d de soldadura debemos aplicar la siguiente fórmula (2):

$$
\mathrm{Ckg}=(\mathrm{Tf}+\mathrm{Te}+\mathrm{Ta}) \mathrm{Ct}+\mathrm{Cc}+\mathrm{Cm}+\mathrm{Ce}
$$


Determinado el costo del kilogramo de metal depositado, podemos medir la productividad de la soldadura en nuestros astilleros y en base a estos datos cada empresa puede tomar las medidas necesarias para aumentar la productividad de la soldadura.

\section{IMPORTANCIA DE LA SOLDADURA EN CONSTRUCCIÓN NAVAL}

La soldadura en la actualidad está íntimamente relacionada con las más importantes actividades industriales: construcción naval, ferroviaria, automovilística, civil, metalúrgica, eléctrica, etc. Existiendo en nuestro país pocas industrias que prescindan de la soldadura como un proceso de producción o de mantenimiento. La soldadura es imprescindible en el campo de la industria naval, pues, en la construcción de las embarcaciones uno de los principales elementos que intervienen es la soldadura, motivo por el cual analizamos la productividad de la misma.

Nuevos sistemas de productividad son los que van a transformar los métodos tradicionales de construcción naval dentro del marco de una estructura organizacional dirigida hacia el producto. Cualquiera sea el término relativo al concepto de productividad global en construcción naval, de alguna manera los trabajos de soldadura incluirán en dicha productividad. Como es de conocimiento en el medio de la construcción naval, los altos porcentajes de costo que intervienen en la soldadura de un buque nos llevan a pesar inmediatamente en la importancia que significa realizar un estudio de la productividad de la soldadura en construcción naval.

Al tener un aumento en la productividad de la soldadura, estamos consiguiendo una disminución en los tiempos de fabricación y hombres/hora, dándonos como resultado una disminución en el costo de la embarcación y el plazo de entrega. Estos dos parámetros de suma importancia para el buen funcionamiento del astillero, construyen las pautas necesarias para optimizar los tiempos de trabajo, el material de aporte entre otros. Pues, de lo contrario resultaría en gastos y tiempos perdidos del astillero. La tecnología de la soldadura es un factor clave que se ha venido desarrollando con el tiempo, alcanzando en la actualidad gran interés por parte del ingeniero y técnico naval, debido a la importante funcional que la soldadura cumple en la estructura de la embarcación.

\section{ELEMENTOS QUE INFLUYEN EN LA PRODUCCIÓN DE LA SOLDADURA CON ELECTRODOS MANUALES}

El rendimiento por fusión (Rf)

Factores que influyen en el rendimiento por fusión

El tipo de electrodo seleccionado

Características tecnológicas de un electrodo

Características económicas de un electrodo

El revestimiento del electrodo

El tipo de planchas y refuerzos

El tipo de unión

La secuencia de soldadura

Los tiempos empleados por el soldador

El estimulo en el soldador

\section{MEDICIÓN DE LA PRODUCTIVIDAD EN ASTILLEROS LOCALES}

\section{Presentación de datos estadísticos}

A continuación vemos algunos datos tomados en la zona de trabajo, valores que permiten tener una idea de cual es la productividad de la soldadura en la industria naval local. Nuestra empresa utiliza como materia prima electrodos de diferentes fabricantes, los tipos de electrodos más usados para soldar la estructura del navío son:

$\mathrm{E}-6011 \quad 1 / 8^{\prime \prime}-5 / 32 ”$

$\mathrm{E}-7018 \quad 1 / 8 "$

\section{Tiempos de fusión de los electrodos}

Las siguientes tablas nos muestran los tiempos que el soldador utiliza para fundir un electrodo, además, la cantidad de metal depositado y la longitud de las sobras. Estos valores servirán para medir el rendimiento de fusión que se menciona en el capítulo anterior, en base a este rendimiento podemos conocer la productividad de la soldadura. Por fines prácticos solo mostraremos la primera de ellas.

\section{Soldadura A}

Tipo de electrodo: E - 7018 1/8"

Amperaje: 130

Posición: horizontal

Soldadura: continua 
Tabla 1. Tiempos de fusión de los electrodos.

\begin{tabular}{llll}
\hline Longitud & Tiempo & Cantidad & Sobra \\
& & & \\
\hline 14.5 & 68 & 1 & 3.0 \\
16.5 & 79 & 1 & 2.3 \\
18.5 & 85 & 1 & 2.0 \\
20.0 & 68 & 1 & 3.3 \\
18.5 & 69 & 1 & 3.0 \\
19.0 & 73 & 1 & 3.1 \\
17.0 & 77 & 1 & 2.6 \\
19.2 & 72 & 1 & 3.4 \\
17.5 & 76 & 1 & 3.6 \\
18.4 & 69 & 1 & 3.7 \\
16.7 & 81 & 1 & 3.0 \\
16.5 & 73 & 1 & 2.8 \\
\hline
\end{tabular}

\section{Número de electrodos por día}

Las siguientes tablas presentan el número de electrodos consumidos durante un día por diferentes soldadores. Valores que fueron tomados aleatoriamente en nuestras empresas y servirán para medir la productividad de la soldadura.

La forma como se toma los datos es: un grupo de empleados día a día mide el consumo de electrodos (peso de cada electrodo), esto es, la cantidad de electrodos que cada soldador consume durante el día de trabajo, como se observa a continuación:

Tabla 2. Cantidad de electrodos astilleros Peruanos.

\begin{tabular}{lllll}
\hline \multirow{3}{*}{ SOLDADOR } & TIPO & E-6011 & E-6011 & E-7018 \\
& & $1 / 8$ & $5 / 32$ & $1 / 8$ \\
& $\phi$ & 3.25 & 4.0 & -25 \\
A Pulg & Lunes & 100 & 20 & - \\
& Martes & - & 105 & - \\
& Miércoles & 95 & - & 80 \\
& Jueves & - & - & - \\
B & Viernes & - & 75 & - \\
& Lunes & 95 & - & 75 \\
& Martes & - & 100 & - \\
& Miércoles & - & - & - \\
& Jueves & 120 & 30 & - \\
C & Viernes & - & - & - \\
& Lunes & 110 & 90 & 85 \\
& Martes & 20 & 15 & - \\
& Miércoles & - & - & 40 \\
\hline
\end{tabular}

Tabla 3. Cantidad de electrodos Astilleros Brasileros

\begin{tabular}{|c|c|c|c|c|}
\hline SOLDADOR & $\begin{array}{l}\text { TIPO } \\
\phi(\mathrm{mm}) \\
\end{array}$ & $\begin{array}{l}\text { E-6011 } \\
3.0 \\
\end{array}$ & $\begin{array}{l}\text { E-6011 } \\
4.0 \\
\end{array}$ & $\begin{array}{l}\text { E-7018 } \\
5.0 \\
\end{array}$ \\
\hline \multirow{7}{*}{ A1 } & Día & \multicolumn{3}{|c|}{ Cantidad de electrodos } \\
\hline & Lunes & 90 & - & 55 \\
\hline & Martes & - & 70 & - \\
\hline & Miércoles & - & - & 65 \\
\hline & Jueves & - & 90 & - \\
\hline & Viernes & 85 & - & - \\
\hline & Lunes & - & 20 & - \\
\hline \multirow{4}{*}{ B1 } & Martes & - & - & 40 \\
\hline & Miércoles & - & 75 & - \\
\hline & Jueves & 60 & - & - \\
\hline & Viernes & - & 90 & - \\
\hline \multirow{5}{*}{$\mathrm{C} 1$} & Lunes & 110 & - & - \\
\hline & Martes & - & - & 60 \\
\hline & Miércoles & - & 80 & - \\
\hline & Jueves & 90 & 50 & - \\
\hline & Viernes & 120 & - & - \\
\hline
\end{tabular}




\section{Cálculo del rendimiento por fusión}

En base a los datos que se muestran en las tablas anteriores, para cada operario (soldador A, B, ..... J) realizamos la sumatoria de la longitud soldada, el tiempo de fusión, la cantidad de material depositado (número de electrodos) y el tamaño de las sobras, como se muestra a continuación:

Tabla 4. Tiempo de fusión para diferentes soldadores.

\begin{tabular}{|l|l|l|l|l|}
\hline Soldador & $\begin{array}{l}\text { Longitud } \\
\text { sold. }\end{array}$ & $\begin{array}{l}\text { Tiempo } \\
\text { de } \\
\text { fusión } \\
\text { (s) }\end{array}$ & $\begin{array}{l}\text { Cantidad } \\
\text { de mat. } \\
\text { (\# elect.) }\end{array}$ & Sobra \\
\hline A & 212.3 & 890 & 12 & 35.8 \\
B & 83.0 & 422 & 7 & 26.2 \\
C & 90.2 & 357 & 6 & 39.3 \\
D & 319.0 & 582 & 9 & 27.1 \\
E & 156.9 & 890 & 13 & 46.5 \\
F & 84.9 & 337 & 6 & 30.3 \\
G & 80.5 & 371 & 5 & 19.6 \\
H & 83.5 & 344 & 5 & 12.5 \\
I & 100.1 & 522 & 9 & 47.8 \\
J & 291.7 & 911 & 16 & 39.7 \\
\hline
\end{tabular}

En función de los tiempos de fusión y la cantidad de metal depositado podemos determinar el rendimiento por fusión, a través de la siguiente fórmula:

$$
\begin{gathered}
\mathrm{Rf}=\frac{\text { cantidad de material }}{\text { tiempo de fusión }}(\mathrm{kg} / \mathrm{hr}) \\
\mathrm{Rf}=\frac{C \times P \times 3600}{T}
\end{gathered}
$$

Donde:

C = cantidad de electrodos consumidos por cada soldador.

$\mathrm{P}=$ peso unitario de cada electrodo, siendo definido de acuerdo al tipo de electrodo utilizado.

$\begin{array}{lc}\text { TIPO } & \text { PESO } \\ \text { E }-60111 / 8 & 0.0256 \mathrm{~kg} \\ \mathrm{E}-70181 / 8 & 0.0333 \mathrm{~kg} .\end{array}$

$\mathrm{T}$ = tiempo de fusión

Además, determinamos el tamaño de la sobra promedio para cada soldador, estos valores pueden observarse en la siguiente tabla.

Tabla 5. Tamaño de la sobra promedio para distintos soldadores.

\begin{tabular}{llllll}
\hline Soldador & $\begin{array}{c}\text { Tipo elec. } \\
\left(\mathbf{1} / \mathbf{8}^{\prime \prime}\right)\end{array}$ & Amp. & Posición de soldar & $\begin{array}{c}\text { Rend. de fusión } \\
(\mathbf{k g} / \mathbf{h r})\end{array}$ & Sobra prom. \\
\hline A & E -7018 & 130 & Horizontal & 1.62 & 2.98 \\
B & E -6011 & 100 & Sob. Cabeza & 1.53 & 3.74 \\
C & E -6011 & 100 & Plana inter. & 1.55 & 6.55 \\
D & E -6011 & 100 & Sob. Cabeza & 1.43 & 3.01 \\
E & E- 7018 & 105 & Sob. Cabeza & 1.75 & 3.58 \\
F & E -6011 & 125 & Plana inter. & 1.64 & 5.05 \\
G & E -6011 & 80 & Plana cont. & 1.24 & 3.92 \\
H & E -6011 & 80 & Plana cont. & 1.34 & 2.50 \\
I & E -7018 & 135 & Sob. Cabeza & 2.07 & 5.31 \\
J & E -6011 & 115 & Sob. Cabeza & 1.62 & 2.51 \\
\hline
\end{tabular}

En esta tabla se observa el rendimiento de fusión de los distintos soldadores, con ciertas características como son: el tipo de electrodo utilizado, amperaje, posición de soldar y la sobra promedio. Valores que permiten analizar la productividad de la soldadura en nuestro medio.

\section{CÁLCULO DE LOS KILOGRAMOS DE METAL DEPOSITADO}

En base a los datos tomados del consumo de electrodos por día en cada soldador, se calcula la media semanal de cada soldador y dividimos por el número de empleados encontrándose la 
producción del grupo por semana. Con estos valores se realiza un gráfico semana por semana o anualmente y se puede hacer comparaciones de la productividad del grupo.
Como los datos obtenidos en las tablas 2 y 3 procedemos a realizar la suma de los electrodos por cada soldador y obtener así la productividad del grupo durante la semana:

Tabla 6. Cantidad de metal depositado en astilleros Peruanos.

\begin{tabular}{|c|c|c|c|c|}
\hline Soldador & $\begin{array}{l}\text { Tipo } \\
\phi(\text { Pulg })\end{array}$ & $\begin{array}{l}E-6011 \\
1 / 8\end{array}$ & $\begin{array}{l}E-6011 \\
5 / 32\end{array}$ & $\begin{array}{l}E-7018 \\
1 / 8\end{array}$ \\
\hline \multirow{5}{*}{ A } & Num. De elect. & 195 & 200 & 80 \\
\hline & Peso unit. (kg) & 0.026 & 0.040 & 0.033 \\
\hline & Peso parc. (kg) & 4.99 & 8.00 & 2.66 \\
\hline & Peso total (kg) & & 15.66 & \\
\hline & Med. x sem (kg) & . & 5.22 & \\
\hline \multirow{5}{*}{ B } & Cantidad de elect. & 215 & 130 & 125 \\
\hline & Peso unit. (kg) & 0.026 & 0.040 & 0.033 \\
\hline & Peso parc. (kg) & 5.51 & 5.20 & 4.16 \\
\hline & Peso total & ………......... & $14.87 \mathrm{~kg}$ & \\
\hline & Méd. x sem & …................. & $4.86 \mathrm{~kg}$ & \\
\hline \multirow{5}{*}{$\mathrm{C}$} & Cant. De elect. & 300 & 105 & 125 \\
\hline & Peso unit. (kg) & 0.026 & 0.040 & 0.033 \\
\hline & Peso parc. $(\mathrm{kg})$ & 7.68 & 4.20 & 4.16 \\
\hline & Peso total & ………….... & $16.04 \mathrm{~kg}$ & \\
\hline & Med. x sem & …………........ & $5.35 \mathrm{~kg}$ & \\
\hline \multicolumn{2}{|l|}{ Media del g } & $\ldots$ & $5.18 \mathrm{~kg}$ & \\
\hline
\end{tabular}

Tabla 7. Cantidad de metal depositado por astilleros Brasileros.

\begin{tabular}{|c|c|c|c|c|}
\hline Soldador & $\begin{array}{l}\text { Tipo } \\
\text { mm }\end{array}$ & $\begin{array}{l}E-6011 \\
3.0\end{array}$ & $\begin{array}{l}E-6011 \\
4.0\end{array}$ & $\begin{array}{l}\text { E - } 7018 \\
5.0\end{array}$ \\
\hline \multirow{5}{*}{ A1 } & Cant. de elect & 175 & 160 & 120 \\
\hline & Peso unit. (kg) & 0.037 & 0.077 & 0.120 \\
\hline & Peso parc. $(\mathrm{kg})$ & 6.47 & 12.32 & 14.40 \\
\hline & Peso total & ........ & $33.19 \mathrm{~kg}$ & \\
\hline & Med. x sem & ........ & $11.06 \mathrm{~kg}$ & \\
\hline \multirow{5}{*}{ B1 } & Cant. de elect. & 60 & 185 & 40 \\
\hline & Peso unit (kg) & 0.037 & 0.077 & 0.120 \\
\hline & Peso parc. $(\mathrm{kg})$ & 2.22 & 14.24 & 4.8 \\
\hline & Peso total & $\cdots$ & $21.26 \mathrm{~kg}$ & \\
\hline & Med. x sem & & $7.09 \mathrm{~kg}$ & \\
\hline \multirow{5}{*}{$\mathrm{C} 1$} & Cant. de elect. & 320 & 130 & 60 \\
\hline & Peso unit (kg) & 0.037 & 0.077 & 0.120 \\
\hline & Peso parc. $(\mathrm{kg})$ & 11.84 & 10.01 & 7.2 \\
\hline & Peso total & & $29.05 \mathrm{~kg}$ & \\
\hline & Med. x sem & & $9.68 \mathrm{~kg}$ & \\
\hline \multicolumn{2}{|c|}{ Media del grupo ............. } & ...... & $3.61 \mathrm{~kg}$ & \\
\hline
\end{tabular}

PROBLEMAS OBSERVADOS EN NUESTROS ASTILLEROS

- Uso de un mismo tipo de electrodo

- Las sobras de los electrodos
- Control de calidad

- Las máquinas de soldar

- Los tiempos empleados por el soldador

- Poco interés del personal en su trabajo

- La seguridad en el área. 


\section{PROPUESTA PARA INCREMENTAR LA PRODUCTIVIDAD DE LA SOLDADURA CON ELECTRODOS MANUALES}

\section{Presentación de la propuesta}

Según el análisis realizado anteriormente, donde fue presentada la productividad en nuestras empresas y al comparar con otros astilleros, se observa que existe diferencias que van a permitir presentar algunas técnicas para incrementar la productividad de la soldadura en base a un efectivo aprovechamiento de los materiales, personal y equipos disponibles en el astillero.

Teniendo especial cuidado en los siguientes aspectos:

- Una correcta elección de los electrodos.

- Reducción de las puntas sobrantes.

- Selección adecuada de refuerzos.

- Selección adecuada de planchas.

- Buena selección de las uniones.

- Reducción de los tiempos auxiliares.

- Aplicación de los estímulos.

\section{Aplicación de un nuevo procedimiento de soldadura}

Los procedimientos de soldadura que se muestra a continuación pueden ser aplicados en nuestro medio, pues, el mayor porcentaje de los trabajos en soldadura son realizados con electrodos manualmente y un pequeño porcentaje con semiautomática.

\section{Soldadura por una sola cara}

En el procedimiento de soldadura convencional, los pasos a seguir para realizar cualquier tipo de unión son los siguientes.

- Soldar por una cara parte de la unión

- Si es posible alterar la pieza, para soldar por la otra cara en posición horizontal, que sería más rentable que soldar en la posición sobre cabeza.

- Esmerilar por la otra cara parte de la primera pasada, hasta encontrar el material completamente limpio y escoria.

- Soldar la segunda cara hasta terminar.
El nuevo procedimiento consiste en colocar por debajo de las planchas en la unión un soporte refractario de cerámica, como se muestra en la figura 1:

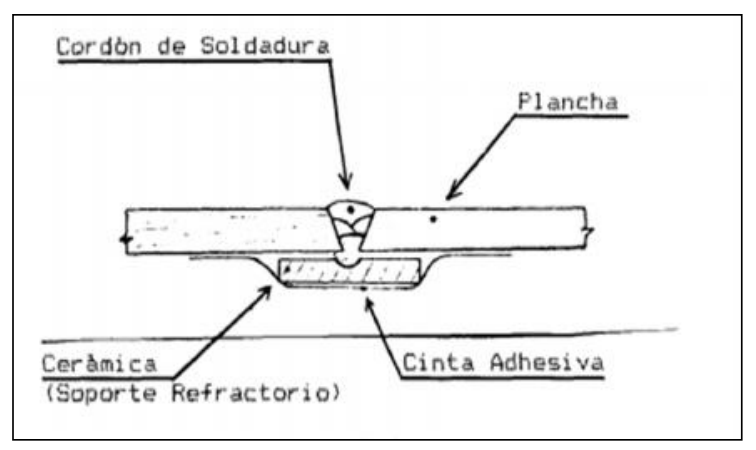

Fig. 1 Soldadura por una sola cara.

De tal manera que el cordón de soldadura aplicado por la cara superior quede realizado con una sola pasada y los pasos quedarían reducidos a.

- Colocar el soporte por la cara contraria a la que vemos a soldar.

- Soldar directamente por la cara superior.

Es decir, que hemos eliminado la operación de volteo o bien la soldadura sobre-cabeza, el resanado luego de pasar el primer cordón de soldadura y la operación de soldar por la otra cara, únicamente se ha aumentado una sola operación que es la de colocar soportes, lo cual se realiza en un tiempo menor que el empleado en las operaciones eliminadas.

Anotamos algunas ventajas de la soldadura por una sola cara o soldadura unilateral:

La principal ventaja es la reducción de los tiempos auxiliares.

- No es necesario el volteo de la plancha

- Evitamos el tiempo de esmerilar el cordón para colocar el segundo cordón de soldadura.

- No es necesario realizar la segunda pasada.

El tipo de soporte que se utiliza en la soldadura por una sola cara con electrodos manuales son soportes rígidos fabricados de dimensiones del 
soporte son $100 \times 20 \times 7 \mathrm{~mm}$ como se muestra en la siguiente figura:

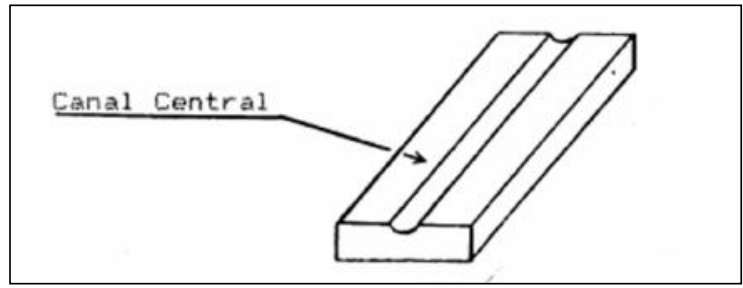

Fig. 2 Chaflán en V.

El chaflán recomendado en este tipo de soldadura es en "V" y el tipo de electrodo recomendado es el de revestimiento básico.

\section{Soldadura por dos electrodos.}

Presentamos este nuevo procedimiento de soldadura como otra alternativa para incrementar el rendimiento del electrodo a través de su diámetro. Al hablar de un incremento en el diámetro del electrodo debemos considerar que, mientras mayor es el diámetro del electrodo la eficacia del revestimiento es menor, siendo necesario una mayor sección del chaflán y por consiguiente mayor cantidad de material de aportación. Por lo antes expuesto se presenta el nuevo procedimiento de soldadura con dos electrodos. El método en sí consiste en la utilización simultánea de dos electrodos conectados al mismo porta electrodo y alimentados por un solo transformador; la característica será que el porta electrodo tendrá dos orificios donde se introducen los electrodos, los mismos que serán ajustados por un sistema de tornillo unido al mango y, un par de seguros que salen de la cabeza del porta electrodo, ayudarán a mantenerse unidos y paralelos durante el proceso de soldadura, como se pueden observar en la siguiente figura:

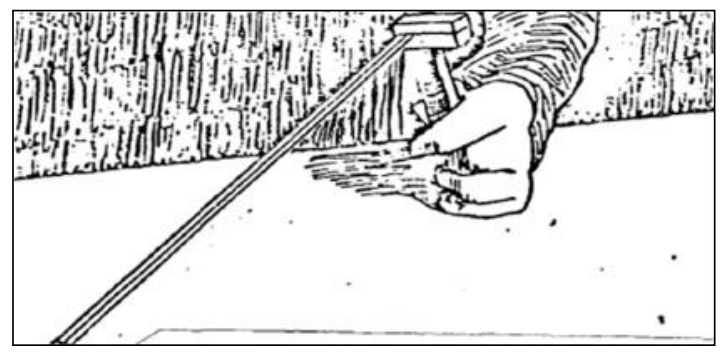

Fig. 3. Soldadura por dos electrodos. La fusión de los electrodos se realiza de tal manera que uno de ellos se funda primero y seguidamente el arco salta al otro electrodo, para fundir durante el mismo tiempo.

El calor del acero de uno de los electrodos favorece al cebado del otro, el tiempo de fusión de cada uno de ellos depende de la intensidad de corriente, del tipo de corriente del tipo de revestimiento y de la longitud del arco.

Con una velocidad de soldadura normal, cada pasada es como si se hiciera con dos pasadas, puesto que, siempre el electrodo anterior deposita sus gotas fundidas sobre el material recién depositado por el primer electrodo.

Las ventajas que ofrece este procedimiento son:

- El tiempo de fusión por dos electrodos es sólo el 20\% más que el procedimiento convencional, obteniendo un ahorro del $40 \%$ aproximadamente en cada electrodo al procedimiento convencional.

- Admite intensidades más altas a igualdad de diámetro de los electrodos respecto al sistema convencional, pues, al tener dos electrodos el diámetro se duplica y el calentamiento debido al amperaje es menor en cada uno.

- El procedimiento genera menos deformación angular en las planchas que soldando con un electrodo, debido a que el número de pasadas es menor.

- Otra ventaja es el tiempo de retirar la escoria, con este procedimiento se reduce a la mitad del empleado en el procedimiento convencional, esto es claro, pues, sólo se hace una pasada y se funden dos electrodos.

- El procedimiento está limitado a lugares bien ventilados o al aire libre, debido a que los gases desprendidos es mayor que al soldar con un electrodo; el manejo del porta electrodo es casi similar a pesar de su mayor peso y tener que controlar un arco en movimiento. 


\section{CONCLUSIONES}

Este trabajo ha sido realizado en base al análisis de la productividad de la soldadura de un astillero nacional, el mismo que fue comparado con una empresa extranjera.

Al realizar la recolección de datos, los cálculos respectivos y obtener consecuentemente los resultados, presentamos algunas técnicas para optimizar la productividad de la soldadura en nuestros astilleros. llegándose a las siguientes conclusiones:

1. Según el análisis seguido para determinar la productividad de la soldadura, podemos concluir que la productividad es baja en nuestras empresas en comparación con empresas extranjeras. Habiendo sido éste el factor que despertó el interés para el desarrollo del presente trabajo, conociendo además que este estudio no ha sido realizado en nuestro país.

Los resultados presentados nos demuestran que existe una diferencia al comparar un grupo de soldadores peruanos con uno brasilero. Sumándose a todo esto los problemas detectados en las visitas realizadas a los astilleros, los mismos que merecen especial atención por parte de la empresa.

Además, esta comparación puede ser realizada dentro de la misma empresa, si dividimos en grupos de trabajo a nuestros soldadores y realizamos comparaciones entre los mismos, la productividad del grupo puede ser determinada mes a mes o también anualmente, permitiendo asi realizar gráficos estadísticos que van a servir para conocer la productividad de la empresa, para una planificación futura mejor.

2. La propuesta y perspectivas presentadas en este trabajo se basa en el análisis de la productividad y los problemas observados en nuestros astilleros; cada técnica o método puede ser aplicado en base a un estudio de factibilidad y con una buena coordinación por parte de los directores de la empresa.
3. En el desarrollo del trabajo se determinó algunos de los factores que inciden en la productividad, existiendo dos que merecen especial atención:

- El personal

- Los soldadores

4. En la última parte de este trabajo se muestra un método para resolver problemas, en vista de que nuestras empresas enfrentan problemas muy diversos los cuales merecen ser analizados y solucionados para el buen desenvolvimiento del astillero, se presenta este método.

\section{RECOMENDACIONES}

Dentro de las recomendaciones a considerar convenientes para trabajos futuros, tenemos las siguientes:

Al realizar la toma de datos, controlar todas las actividades de un soldador durante un día normal de actividades, para tener una idea más amplia de todos los tiempos utilizados por el soldador. Además tratar de tomar una mayor cantidad de datos.

Llevar a la práctica los nuevos procedimientos de soldadura, para lo cual será necesario conseguir el financiamiento.

Para garantizar una mayor productividad en nuestros astilleros mantengamos una buena relación con el personal, escuchando sus planteamientos y sugerencias, pues, van a ayudar mucho en el desarrollo de la empresa.

\section{REFERENCIAS}

1. Almeida Calegare, J., "Un método para el aumento de la productividad, I Congreso Panamericano de Ingeniería Naval, España (1978).

2. Gómez Moreno, "Sesiones de Productividad de la Soldadura en Construcción Naval", Escuela Técnica Superior de Ingenieros Navales, Madrid, (1992). 
3. Toshie Okumura, Taniguchi Celio: "Engenhieria de Soldagem y Aplicaciones", Sao-Paulo, Brasil (1998).

Ingeniería Portuaria, Guayaquil, Ecuador (2005).

4. Yarin, "Inspección Visual e Importancia de la Soldadura en el Proceso de Construcción de Estructuras Navales", XIX Congreso Panamericano de Ingeniería Naval, Transporte Marítimo e

Correspondencia: hegch16@yahoo.com.mx 